\title{
Humanización del acto de la retroalimentación en la educación virtual
}

María Elsy Sepúlveda-Romero

Universidad EAN

mesepulveda@universidadean.edu.co

ORCID: https://orcid.org/0000-0002-4388-9589

DOI: https://doi.org/10.21158/2357514x.v7.n1.2019.2326

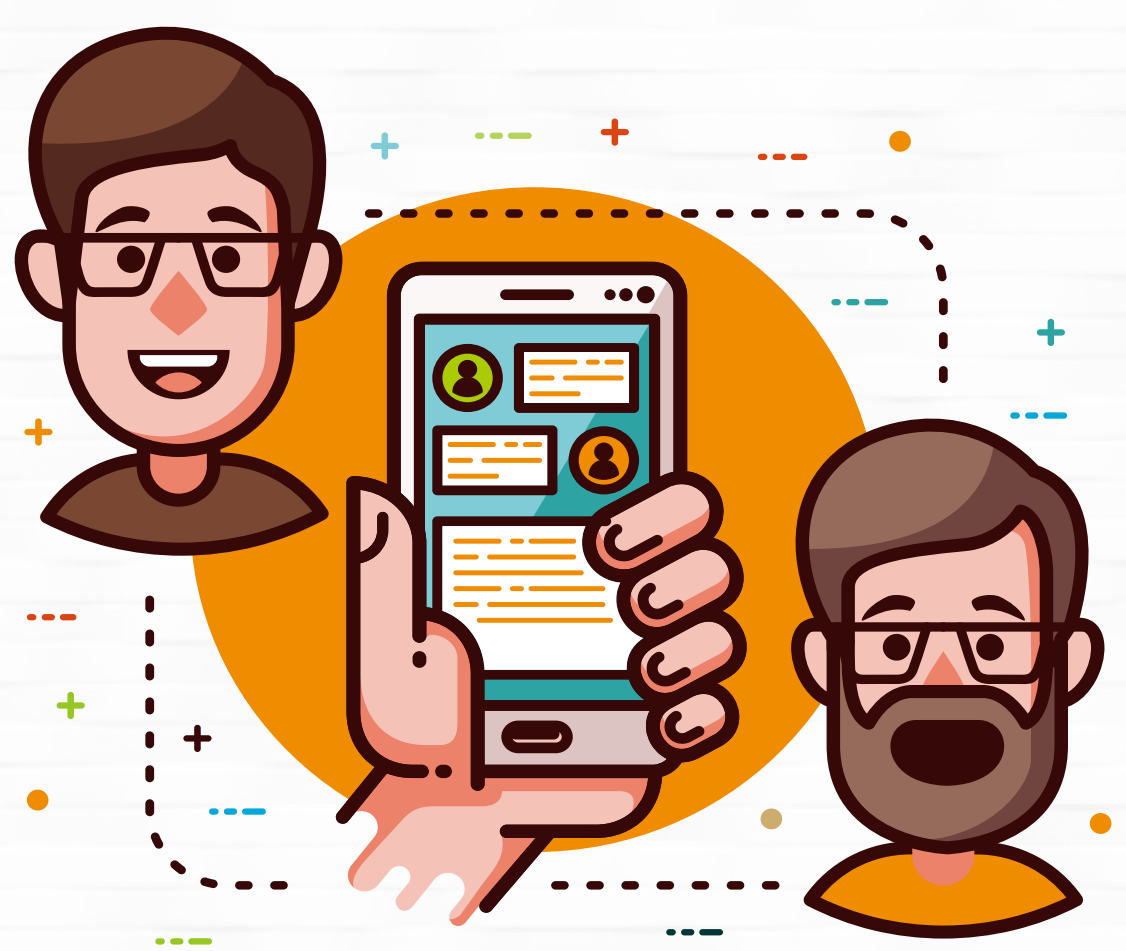

Cómo citar este artículo: Sepúlveda-Romero, M. E. (2019). Humanización del acto de la retroalimentación en la educación virtual. Revista Virtu@lmente, 7(1), 95-115. DOI: https://doi.org/10.21158/2357514x.v7.n1.2019.2326

Fecha de recepción: 26 de junio de 2018

Fecha de aprobación: 16 de octubre de 2019 


\section{RESUMEN}

Con el progreso de las TIC, nace en medio de un proceso histórico la fusión entre la educación tradicional con la tecnología, dando como resultado la educación virtual, por ende, el acto de la retroalimentación, que no sólo se utiliza para revisar un trabajo, detectar errores o señalar aciertos, sino para orientar, apoyar y estimular al estudiante en su posterior aprendizaje, de ahí, que la finalidad principal de este artículo es identificar el proceso de la humanización del acto de la retroalimentación en la educación virtual.

Para alcanzar el propósito de la investigación se establecieron cuatro objetivos específicos: precisar los conceptos de humanización, retroalimentación y educación virtual; analizar el acto de la retroalimentación como instrumento en la educación virtual; enunciar las principales herramientas utilizadas en la retroalimentación en la educación virtual, y por último, establecer cómo se genera la humanización del acto de la retroalimentación. En el aspecto metodológico lo que se hizo fue revisar y analizar literatura de investigaciones y estudios realizados por diferentes autores tanto nacionales como extranjeros. Entre los hallazgos encontrados se destaca que el proceso de la humanización del acto de la retroalimentación en la educación virtual se refleja en el seguimiento que se hace al estudiante, el conocimiento que se tiene de sus dificultades, el progreso que se da a su aprendizaje y en la identificación de su contexto.

Palabras clave: educación virtual; retroalimentación en la educación; educación virtual y humanización; proceso de aprendizaje; herramientas para retroalimentación; herramientas de aprendizaje. 


\section{Humanization of the act of feedback in virtual education}

\section{ABSTRACT}

With the progress of the Information and Communication Technologies (ICT), the fusion between traditional education and technology is born in the middle of a historical process, resulting in virtual education, therefore, the act of feedback, which is not only used to review a work, detect errors, or point to successes, but to guide, support, and stimulate the students in their subsequent learning, hence, the main purpose of this article is to identify the process of humanization of the act of feedback in virtual education.

In order to achieve the purpose of the investigation, four specific objectives were established: to specify the concepts of humanization, feedback, and virtual education; to analyze the act of feedback as an instrument in virtual education; to state the main tools that are used in feedback in virtual education and finally, to establish how the humanization of the act of feedback is generated. In the methodological aspect, what was done was to review and analyze research literature and studies carried out by different national and foreign authors. Among the findings, we can highlight that the process of humanization of the act of feedback in virtual education, is reflected in the follow-up that is made to the student, the knowledge that is had of their difficulties, the progress that is given to their learning and in the identification of its context.

Keywords: virtual education; feedback in education; virtual education and humanization; learning process; feedback tools; learning tools. 


\section{Humanização do ato de retroalimentação na educação virtual}

\section{RESUMO}

Com o progresso das TIC, a fusão entre educação tradicional e tecnologia nasce no meio de um processo histórico, resultando em educação virtual, portanto, o ato de retroalimentar, que não é usado apenas para revisar um trabalho, detectar erros ou apontar para sucessos, mas para orientar, apoiar e estimular o aluno em sua aprendizagem subsequente, portanto, o principal objetivo deste artigo é identificar o processo de humanização do ato de retroalimentação na educação virtual.

Para atingir o objetivo da pesquisa quatro objetivos específicos foram estabelecidos: especificar os conceitos de humanização, retroalimentação e educação virtual; analisar o ato da retroalimentação como um instrumento na educação virtual; declarar as principais ferramentas usadas na retroalimentação na educação virtual e, finalmente, estabelecer como a humanização do ato de retroalimentar é gerada.

Quanto ao aspecto metodológico, analisou-se a literatura de pesquisas e estudos realizados por diferentes autores nacionais e estrangeiros. Como resultado constatou-se que o processo de humanização do ato de retroalimentar na educação virtual se reflete no acompanhamento que é feito ao aluno, no conhecimento que se tem de suas dificuldades, no progresso que é dado à sua aprendizagem e na identificação de seu contexto.

Palavras-chave: educação virtual; retroalimentação na educação; educação virtual e humanização; processo de aprendizagem; ferramentas de retroalimentação; ferramentas de aprendizagem. 


\section{Humanisation de l'acte de rétroaction en éducation virtuelle}

\section{RÉSUMÉ}

Grâce aux progrès des TIC, la fusion entre éducation traditionnelle et technologie aboutit à une éducation virtuelle et à l'acte de rétroaction (ou de retour d'information) qui n'est pas seulement utilisé pour évaluer une copie, détecter des erreurs ou mettre en lumière des points forts, mais pour guider, aider et stimuler l'apprenant dans son parcours d'apprentissage. L'objet principal de cet article abordera l'identification du processus d'humanisation de la rétroaction en éducation virtuelle.

Pour atteindre ce dessein, quatre objectifs spécifiques ont été définis: préciser les concepts d'humanisation, de retour d'information et d'éducation virtuelle; analyser l'acte de rétroaction en tant qu'instrument d'éducation virtuelle; énoncer les principaux outils utilisés lors de la rétroaction et enfin déterminer comment humaniser ce retour d'information. Sur le plan méthodologique, une analyse de la littérature des recherches et investigations réalisées par différents auteurs nationaux et étrangers a été effectuée, avec en conclusion, que le processus d'humanisation de l'acte de rétroaction en éducation virtuelle se reflète dans le suivi apporté aux étudiant, dans la connaissance de leurs difficultés, des progrès accomplis et de l'identification de leurs contextes personnels particuliers.

Mots-clés: éducation virtuelle; retour d'information dans l'éducation; éducation virtuelle et humanisation; processus d'apprentissage; outils de rétroaction; outils d'apprentissage. 


\section{Introducción}

En el siglo $X X$, la educación presencial se consideraba la única forma de lograr una formación que permitiera dar las habilidades y los conocimientos necesarios; sin embargo, la evolución de la tecnología modificó este paradigma. Por el desarrollo que ha tenido la tecnología de la información y la comunicación (TIC), los medios de comunicación, interacción y adquisición de nuevos aprendizajes han evolucionado a través de los entornos virtuales, lo que ha generado en el hombre un cambio significativo en el concepto y la vivencia de su propia realidad, que lo ha llevado a observar lo que ha ganado con la tecnología, pero también lo que ha perdido con ella, por ejemplo, el contacto directo o real con las personas, socializar, la relación que se tiene cara a cara y los sentimientos y expresiones espontáneas, ausencias que no aportan a lo humano (Criollo, 2007).

No obstante, a partir de ese progreso de las TIC, nació la fusión entre la educación tradicional con la tecnología en medio de un proceso histórico que dio como resultado la educación virtual, que desencadenó que los procesos educativos se modificaran, porque hubo un desarrollo de las herramientas en la web y por los cambios en las condiciones sociales, de ahí que la educación virtual presentó algunas ventajas y desventajas. Entre las ventajas, se dio lugar a un nuevo escenario que configura un aprendizaje diferente al que existía de manera presencial, lo que ha generado que tanto el docente como el estudiante potencien nuevas competencias en torno a los nuevos desafíos que propone la educación virtual (Pérez, Subirá y Guitert, 2011).
Entre las desventajas, las hay de nivel intelectual, epistemológico y comunicacional. Al respecto, Ralón, Vieta y Vásquez (2004) manifestaron que es virtualmente imposible en el formato online que el estudiante pueda discutir con desafiar las ideas del docente o que las expresiones faciales y no verbales -vitales en el proceso comunicativo-sean totalmente ilegibles vía internet. También expresaron que muchos docentes se han quedado únicamente en lo instruccional, organizacional, operativo y en las competencias, sin un criterio que enfatice en que este debe acercarse al estudiante para utilizar todos los medios con los que dispone la tecnología en la actualidad (Criollo, 2007).

Debido a estas circunstancias, se presenta el hecho de que los ambientes virtuales se han constituido en espacios humanos que requieren ser identificados en toda su riqueza y complejidad, con la finalidad de comprenderlos para mejorarlos (Cardona, 2008) pues, en esta modalidad, el docente debe asumir nuevas funciones $y$, por ende, nuevos roles (Criollo, 2007). Dentro de este contexto, se realiza la presente investigación, con el objetivo de identificar el proceso de la humanización del acto de la retroalimentación en la educación virtual.

Para el desarrollo, se elaboró en cuatro secciones, así: a) se precisan los conceptos de humanización, retroalimentación y educación virtual; b) se analiza el acto de la retroalimentación como instrumento en la educación virtual, c) se enuncian las 
principales herramientas utilizadas en la retroalimentación en la educación virtual, y d) se establece cómo se genera la humanización del acto de la retroalimentación.

\section{Generalidades sobre humanización, retroalimentación y educación virtual}

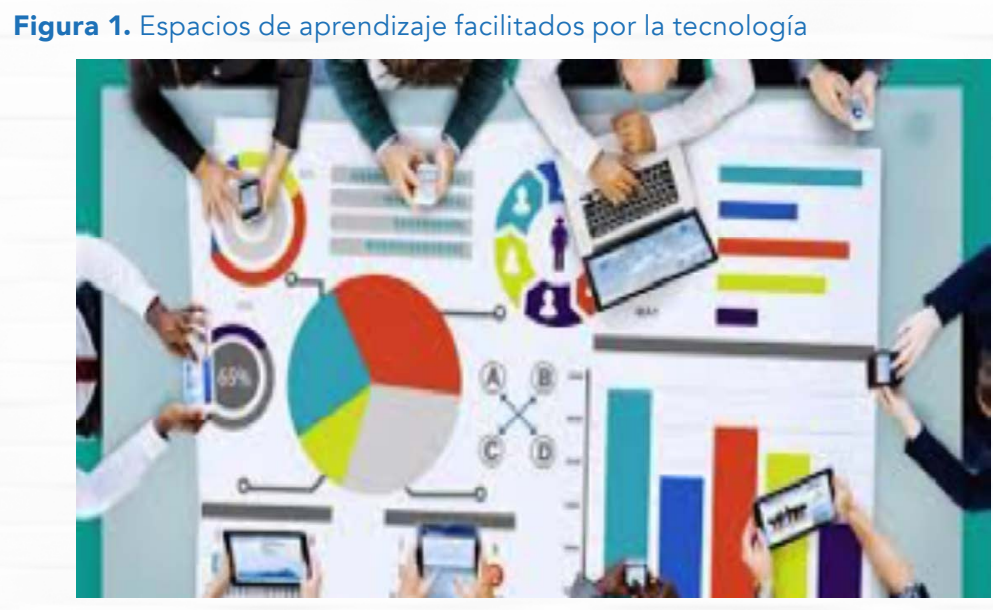

Fuente. The flipped classroom, 2017.

Esta primera sección presenta un acercamiento conceptual de carácter general de los términos humanización, retroalimentación y educación virtual, con el fin de tener claridad sobre dichos términos, los cuales son el soporte de esta investigación.

\subsection{Humanización}

Para Lozada et al. (2013), la humanización se refiere a los procesos integrales de formación donde al hombre y a la mujer se miran con dignidad y respeto en todas sus dimensiones: intelectual, afectiva, social y cultural. Es recuperar la individualidad que hace único a cada ser humano.

Vista desde otra perspectiva, Carbonell y Hortolà (2013) definieron el concepto humanizarse, como «el proceso de singularidad evolutiva que nos ha llevado a la conciencia operativa» (p. 10), y a la humanización como el surgimiento de la inteligencia operativa, resultado de su socialización. También expresaron que es la adquisición de la capacidad de pensar sobre nuestra inteligencia, de poder entender el proceso de la vida, así como de adaptarse al entorno a través del conocimiento, la tecnología y el pensamiento.

\subsection{Retroalimentación}

En inglés, feedback. Papic, Rittershaussen y Rodríguez (1987) manifestaron que proviene de la cibernética y la tecnología general de sistemas, que existe un emisor y un receptor con mutua interdependencia y niveles de interacción, en donde el emisor verifica que los mensajes transmitidos sean asimilados por el receptor, pues, en cibernética, los elementos que conforman el feedback deben mantener una clara comunicación entre sí. De este modo, se desarrollan interrelaciones coherentes, debido a que constituye una alimentación al revés que actúa retroactivamente como mecanismo de control, tanto positivo como negativo; su misión es amplificar y corregir la desviación de un sistema para mantenerlo en equilibrio. 
Entendida así la retroalimentación, en el campo educativo permite que el docente y el estudiante tengan una comunicación interpersonal no lineal, sino de forma circular, en donde se toma en cuenta la información sobre las acciones pasadas para decidir sobre lo futuro (Cabinal, 2008, citado por Martínez, 2011), es decir, que la retroalimentación se constituye en una labor que consiste en comunicarle al estudiante su desempeño, de acuerdo a la etapa formativa y al desarrollo personal de este (Martínez, 2011).

Por otro lado, Durante y Sánchez (2006) afirmaron que la retroalimentación es un proceso constructivo de formación, cuya finalidad no es solo evaluar o enjuiciar a quien realiza un procedimiento, sino que señala fortalezas y debilidades en torno a una actividad realizada, con el fin de estimular cambios tanto en el estudiante como en el docente, a quien le informaran sobre la efectividad de su interacción comunicativa con el alumno.

Analizando otros autores, se tiene que Unigarro (2001) se refirió a un proceso relacionado con la información que el docente le suministra al estudiante sobre el logro de sus metas de formación propuestas, es decir, es el feedback que toda persona espera con ansiedad durante su proceso educativo. Mogollón (2004), por su parte, la consideró como una comunicación de forma descriptiva y continua, que le facilita información al estudiante sobre sus avances desde el inicio hasta el final del desarrollo del proceso de enseñanza aprendizaje y Scagnoli (2001) argumentó que el aula virtual debe proveer el espacio para que los estudiantes reciban o envíen sus evaluaciones al docente, para que este pueda leer, corregir y devolver por el mismo medio.

\subsection{Educación virtual}

Parra (2005) indicó que la educación virtual se posiciona en todo el mundo como una alternativa útil para esa acelerada movilidad en que se halla la población, pero que no tiene acuerdos entre sus gestores y usuarios de su verdadero significado.

Para conceptualizar la palabra «educación virtual», es importante primero definir lo que quiere decir «virtual». El Diccionario de la Real Academia Española (2002) dice «que tiene virtud para producir un efecto, aunque no lo produce de presente, frecuentemente en oposición, a efectivo o real, implícito, tácito, que tiene existencia aparente y no real» y el Diccionario de Uso del Español de María Moliner (1998), citada por Parra (2005), define la palabra virtual así: «Se aplica a lo que tiene existencia aparente. Se dice sobretodo de las imágenes, sonidos o sensaciones en general que, creados por medios informáticos, producen en quien los recibe, ilusión de realidad: realidad virtual» (p. 11).

De manera que, uniendo las dos palabras, para Rubio (2002) la educación virtual o e-learning es una forma de educación a distancia que surgió con el desarrollo de la tecnología de la información y del internet, y se basa en aprovechar la facilidad de distribución de materiales formativos y herramientas de comunicación para crear un entorno para el aprendizaje.

Cardona (2008) explicó la educación virtual como aquellos ambientes educativos que están mediados por las TIC y que se convierten en un escenario cultural contemporáneo, donde hacen aparición todos aquellos rasgos de comportamiento 
que caracterizan a la cultura humana. Por su parte, Pedraza (2003), citado por Ralón, Vieta y Vásquez de Prada (2003), con respecto al entorno virtual, aseveró que se caracteriza porque ha ampliado el acceso a la educación, ha promovido el aprendizaje activo, el colaborativo y el trabajo en grupo, crea comunidades de aprendizaje, está centrado en el estudiante y, por último, ha hecho los roles tradicionales de enseñanza aprendizaje de forma más fluida.

Para el Ministerio de Educación (2009), es aquella en donde no es necesario que se conjuguen el cuerpo, el tiempo y el espacio para lograr establecer un diálogo o una experiencia de aprendizaje, pero que sí es posible establecer una relación interpersonal de carácter educativo.

\section{La retroalimentación como instrumento en la educación virtual}

En esta sección, se considera el acto de la retroalimentación como instrumento que sirve para el aprendizaje en la educación en el ámbito virtual, analizándola desde la diferencia que existe entre la evaluación, la retroalimentación y desde sus características.

Para comenzar, se podría decir que la educación virtual rompió con algunos paradigmas, pasando de enseñar a aprender y de evaluar a retroalimentar, en donde el aprender llevó al docente y al estudiante a desarrollar habilidades y competencias en un mismo entorno virtual de aprendizaje y la retroalimentación exigió del docente buena actitud y aptitud para asumir el compromiso de revisar, evaluar y reorientar el desempeño del estudiante hacia el mejoramiento de su rendimiento académico.

\subsection{Evaluar vs. retroalimentar}

Suele confundirse el proceso de la retroalimentación con la evaluación. Para Martínez (2011) evaluar significa «juzgar el desempeño del educando» (p. 27), por lo tanto, se presentan algunas diferencias entre los dos conceptos, así:

Tabla 1. Diferencias entre la evaluación y la retroalimentación

\begin{tabular}{|c|c|}
\hline La evaluación & La retroalimentación \\
\hline $\begin{array}{c}\text { Se establece como un } \\
\text { juicio de valor. }\end{array}$ & $\begin{array}{c}\text { Constituye el retorno de información } \\
\text { sobre un proceso de aprendizaje, acorde } \\
\text { a metas prefijadas, es decir, que describe } \\
\text { información. }\end{array}$ \\
\hline $\begin{array}{c}\text { Constituye el final de un } \\
\text { proceso en conocimiento } \\
\text { y meta, es decir, es } \\
\text { unidireccional. }\end{array}$ & $\begin{array}{c}\text { Es un instrumento que informa al } \\
\text { estudiante sobre su proceso de } \\
\text { aprendizaje para facilitar los cambios } \\
\text { necesarios, es multidireccional. }\end{array}$ \\
\hline $\begin{array}{c}\text { Puede generar reacciones } \\
\text { defensivas, porque se } \\
\text { centra en calificar, debido } \\
\text { a que debe cumplir con las } \\
\text { demandas institucionales. }\end{array}$ & $\begin{array}{c}\text { Es recibida con agrado, se le presta } \\
\text { atención y se agradece, a pesar de haber } \\
\text { aceptación tornándose en autorreflexiva. }\end{array}$ \\
\hline $\begin{array}{c}\text { Está centrada en el sistema } \\
\text { que le exige a emitir un } \\
\text { juicio, es decir, que juzga. }\end{array}$ & $\begin{array}{c}\text { Está centrado en el aprendizaje y sus } \\
\text { procesos, es decir, que asesora. }\end{array}$ \\
\hline $\begin{array}{c}\text { Es medición o } \\
\text { diagnóstico de una tarea } \\
\text { desempeñada. }\end{array}$ & $\begin{array}{c}\text { Es mediación para ejercer la evaluación } \\
\text { con justicia y aportar al estudiante una } \\
\text { manera de crecer. }\end{array}$ \\
\hline
\end{tabular}




\begin{tabular}{|c|c|}
\hline $\begin{array}{c}\text { Es una expresión } \\
\text { «simbólica» en donde el } \\
\text { estudiante extrae poca } \\
\text { información, porque } \\
\text { termina con un dígito, pues } \\
\text { utiliza expresiones como } \\
\text { «suficiente», «insuficiente», } \\
\text { etc. }\end{array}$ & $\begin{array}{c}\text { Es efectiva cuando cierra la brecha entre } \\
\text { el nivel actual de un trabajo y el nivel de } \\
\text { referencia de un sistema de parámetros, } \\
\text { implicando así una acción concreta. }\end{array}$ \\
\hline $\begin{array}{c}\text { La información que } \\
\text { ofrece es focalizada } \\
\text { retrospectivamente, } \\
\text { cuestionándose si lo hizo } \\
\text { bien o lo hizo mal. }\end{array}$ & $\begin{array}{c}\text { La información que ofrece es focalizada } \\
\text { prospectivamente, visualizándose. ¿Qué } \\
\text { hacer ahora? }\end{array}$ \\
\hline
\end{tabular}

Fuente. Martínez, 2011, p. 6.

Lo anterior conduce a pensar que la evaluación puede ser vista de forma negativa en el proceso educativo, por las características que posee, como son: emite un juicio de valor, pues tiene como finalidad la aprobación; es unidireccional, porque solo se tienen en cuenta los parámetros establecidos por el docente; solo se limita a calificar, se sujeta a un dato cuantitativo, es decir, a una nota; juzga, porque se le da más importancia a debilidades y errores que a los logros; diagnostica, solo lleva a la respuesta correcta, dejando de lado el proceso cognitivo que realizó el estudiante para elaborar la respuesta; se expresa simbólicamente, en muchos casos se utilizan como forma de evaluar letras como A, D y otras; es retrospectiva, porque no le da la oportunidad de mejorar al estudiante, por motivo de que los resultados se toman como definitivos.

Mientras que la retroalimentación se observa de manera positiva, debido a que es informativa; multidireccional, pues en ella intervienen simultáneamente el docente y el estudiante; autorreflexiva, dado que le permite al estudiante confirmar o corregir su desempeño; asesora, pues se encamina al estudiante en la dirección correcta; hace crecer, aumenta el rendimiento del estudiante; hace referencia a una acción concreta y sobre todo es prospectiva, debido a que encamina al estudiante a la meta propuesta, pero si se complementan la evaluación y la retroalimentación, pueden ser favorables para el aprendizaje del estudiante en la educación virtual.

De manera que la realización de la anterior tabla es importante porque muestra las características de cada una, con lo cual se refleja que la retroalimentación le aporta más al estudiante en el proceso educativo y, específicamente, en su aprendizaje. Al respecto de la retroalimentación evaluativa, Maldonado (2009) establece unos criterios que se dividen en cinco clases: a) directa, dirigida a un miembro del grupo en particular, b) descriptiva, que expresa en forma detallada las características de la conducta a la que está referida, c) específica, referida a una situación determinada en un momento preciso, d) inmediata, referida a una situación próxima muy cercana, y e) la que tiene expresión emocional, demuestra la emoción y el sentimiento que acompaña la comunicación. Estas clases no se excluyen entre sí, sino que se complementan, por lo tanto, deben estar presentes siempre en el proceso formativo.

Entonces, la retroalimentación o el feedback están relacionadas con la evaluación y es en ella, pero de forma más extensa, en donde se devuelve la información procesada para reforzar los conocimientos adquiridos, para ir más allá en el proceso formativo, que termina cuando el estudiante ha llegado al conocimiento meta propuesto en la evalua- 
ción. Ello supone que la evaluación esté ligada al aprendizaje (Maldonado, 2009), lo cual coincide con lo que afirmaron Pérez y Salas (2016), que la evaluación y el aprendizaje son dos conceptos que se encuentran interrelacionados con la retroalimentación, porque el aprendizaje es el propósito de todo cuanto se persigue, cuando hay una adecuada retroalimentación en el proceso educativo y la retroalimentación es el eje para promover el aprendizaje, debido a que involucra al docente y al estudiante en las metas que deben alcanzar, que les permite tomar decisiones para mejorar dicho proceso, es decir, el docente proporciona al estudiante las estrategias necesarias para mejorar su propio aprendizaje (Amaranti, 2010).

\subsection{Características de la retroalimentación}

Para que la retroalimentación esté completa, incluye tres conceptos fundamentales: el feed up, que significa hacia dónde va el alumno; el feedback, que le ayuda al alumno a darse cuenta cómo se está desempeñando, y el feedforward, que responde a los interrogantes sobre, ¿qué sigue ahora? o ¿cómo puede mejorar para la siguiente actividad? (Alvarado, 2014).

Osorio y López (2014) opinaron que la retroalimentación favorece el hecho de que el estudiante crea en lo que está haciendo y se detenga en un momento dado a interiorizar sus resultados, para que así pueda deducir que existen algunas formas adecuadas para realizar las tareas en cada uno de los ámbitos, es decir, lo que caracteriza la retroalimentación es la confianza en sí mismo que genera al estudiante.
Para Cruz (2008), una buena retroalimentación incluye varios aspectos, son los siguientes: ayuda a aclarar lo que es un buen desempeño; facilita el desarrollo de la reflexión y autoevaluación del aprendizaje; entrega al estudiante información de calidad sobre su aprendizaje; promueve el diálogo entre el docente y el estudiante; fomenta la autoestima y las creencias motivacionales positivas; brinda oportunidades para cerrar la brecha entre el desempeño dado y el deseado y provee información al docente, que puede ser utilizada para modificar la enseñanza.

En su análisis a las posturas de diferentes autores (Gitman y MacDaniel, 2008; Mosley, Megginson y Pietri, 2005; Durante y Sánchez, 2006; Ávila, 2009; Fornells, Juliá, Arnau y Martínez, 2008), Martínez (2011) dedujo que la retroalimentación en la educación virtual tiene que ver con el cumplimiento de unas metas que se concretan en el aprendizaje del currículo en desarrollo, ellas son:

a) Ser necesaria, pues le ayuda al estudiante a evaluar su desempeño real.

b) Ser pronta y oportuna.

c) Incidir en tres planos de motivación: cómo conducirse, monto del esfuerzo y grado de persistencia.

d) Aportar información que refuerce el proceso de aprendizaje. 
e) Detectar problemáticas de aprendizaje en forma oportuna.

f) Constituirse en un espejo que permita alinear lo que se está haciendo con la realidad para mejorar.

g) Generar procesos de autoconfianza.

En principio, si se encuentran ausentes estas metas, el estudiante no tendrá información sobre su desempeño, ya sea en sus puntos débiles o fuertes, con el fin de que el docente pueda corregir los errores y así pueda reforzar la buena práctica.

Maldonado (2009) propuso que para que la retroalimentación sea efectiva en la educación virtual y que el proceso de aprendizaje sea mejor, debe reunir las siguientes condiciones:

a) Señalar y corregir inconsistencias del proceso gradual de conocimiento.

b) Reforzar el aprendizaje.

c) Tener precisión en la información que se necesita ajustar.

d) Tener continuidad para mejorar el desempeño del estudiante.

e) Plantear cómo proceder en la estrategia formativa posterior.
Igualmente, Mogollón (2004) y Villardón (2006), apoyados en diversos autores, plantearon que la retroalimentación debe cumplir con unas condiciones mínimas en la educación virtual, así:

a) Ha de orientarse a la conducta y no a la persona.

b) El diseño del curso debe establecer las bases de la retroalimentación con demandas específicas, previamente conocidas por el alumno.

c) Debe ser rápida y oportuna.

d) Debe existir un compromiso del docente con la retroalimentación emitida.

e) Debe existir un cumplimiento de fechas límites para la entrega de las retroalimentaciones.

f) Aunque habla del pasado, se orienta hacia el futuro y debe prestar atención en los objetivos pretendidos.

g) Debe generarse una especie de diálogo por escrito, favoreciendo procesos de invitación para objetivar su propia producción.

h) Debe ser continua, para que tenga seguimiento y control.

i) Ha de estimular, orientar y hacer efectivo el aprendizaje independiente. 
En consecuencia, una retroalimentación de buena calidad reúne algunos atributos como son: exige ser directa -personal-, descriptiva -detallada-, específica -clara sobre algo concreto-, inmediata $-u$ oportuna- y con expresión emocional -que revele interés del docente por el progreso del estudiante-. Solo así, estimulará el desarrollo de la reflexión y los procesos de autoevaluación (Mogollón, 2004; Villardón, 2006).

De las anteriores características, se deduce que la retroalimentación es el principal mecanismo de aprendizaje, debido a que en la educación virtual, las actividades realizadas son documentos escritos para ser leídos, esto hace que muestre una lectura seria por parte del docente, quien está obligado a dejar evidencia, lo que constituye un acto de comunicación entre el docente y el estudiante, de ahí que el vínculo que se da entre las dos partes en un ambiente virtual se fortalece a través de adecuados procesos de retroalimentación, la cual debe estar regulada para que la formación virtual cumpla las metas de una verdadera educación, porque de ella depende que el estudiante crea y tenga confianza en sí mismo, le genere reflexión, alimente su autoestima, lo motive y le refuerce y acreciente su aprendizaje.

\section{Herramientas virtuales utiliza- das para la retroalimentación}

Figura 2. Interconexión en la educación

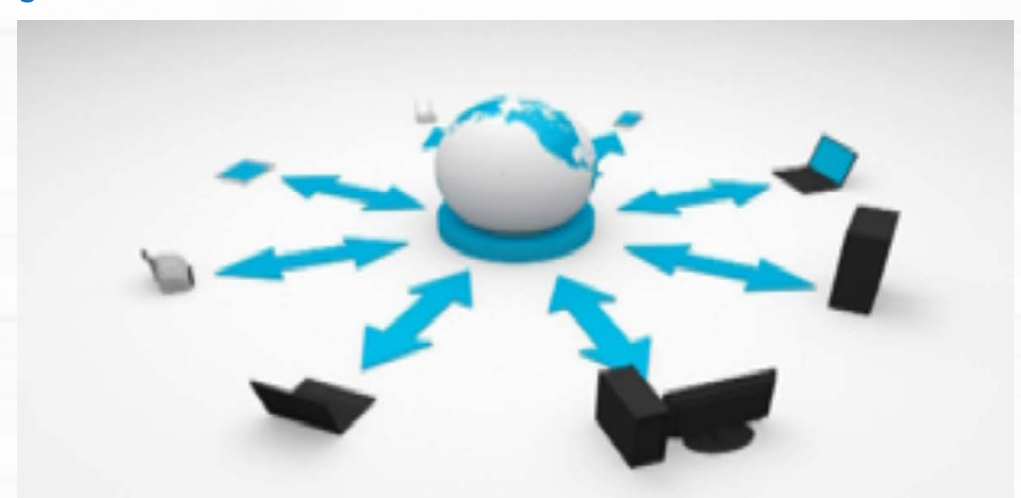

Fuente. E-learning Masters, 2018.

Las siguientes son herramientas virtuales que facilitan el aprendizaje y que son utilizadas para la retroalimentación: 
Tabla 2. Herramientas virtuales utilizadas para la retroalimentación

\begin{tabular}{|c|c|c|}
\hline Herramientas & Concepto & Uso didáctico \\
\hline $\begin{array}{l}\text { El correo } \\
\text { electrónico }\end{array}$ & $\begin{array}{l}\text { Es una herramienta de comunicación asincrónica que envía y recibe informa- } \\
\text { ción, de interés específico para un grupo de usuarios. }\end{array}$ & $\begin{array}{l}\text { Permite aprendizajes cooperativos, colaborativos y significativos, } \\
\text { se convierte en un mediador pedagógico. }\end{array}$ \\
\hline $\begin{array}{l}\text { El tablero } \\
\text { compartido } \\
\text {-pizarra- }\end{array}$ & $\begin{array}{l}\text { Es una herramienta sincrónica que permite introducir textos, dibujar objetos, } \\
\text { insertar gráficas y hacer modificaciones. }\end{array}$ & $\begin{array}{l}\text { Está estrechamente relacionado con el chat, por ser una herra- } \\
\text { mienta sincrónica, resulta especialmente útil para dibujar y visua- } \\
\text { lizar diagramas en tiempo real. }\end{array}$ \\
\hline $\begin{array}{l}\text { Foros de } \\
\text { debate o grupos } \\
\text { de discusión }\end{array}$ & $\begin{array}{l}\text { Son espacios donde los mensajes se pueden poner a disposición de todos los } \\
\text { matriculados en un curso virtual, es de carácter asincrónico. }\end{array}$ & $\begin{array}{l}\text { Sirve para discutir casos de estudio, problemas u otras temáticas } \\
\text { de interés para el grupo. Al clausurarse el foro, el docente } \\
\text { encarga al estudiante la realización de una relatoría que recoja las } \\
\text { conclusiones, que debe enviarse a todos los estudiantes, como } \\
\text { un producto elaborado de trabajo colaborativo y cooperativo. }\end{array}$ \\
\hline $\begin{array}{l}\text { Página web del } \\
\text { estudiante }\end{array}$ & $\begin{array}{l}\text { Las plataformas (LMS) presentan esta herramienta que permite al estudiante } \\
\text { elaborar un sitio web personal. }\end{array}$ & $\begin{array}{l}\text { Posibilita el desarrollo de trabajo cooperativo, la evaluación } \\
\text { formativa, la interacción grupal y la creación de comunidades } \\
\text { virtuales de aprendizaje. }\end{array}$ \\
\hline $\begin{array}{l}\text { Presentaciones de los } \\
\text { estudiantes -trabajos en } \\
\text { equipo- }\end{array}$ & $\begin{array}{l}\text { Permite a los estudiantes realizar presentaciones o exposiciones sobre temá- } \\
\text { ticas correspondientes al curso. }\end{array}$ & $\begin{array}{l}\text { Permite explicar conceptos en pocas palabras, también posibilita } \\
\text { el desarrollo de trabajo cooperativo, la evaluación formativa, la } \\
\text { interacción grupal y la creación de comunidades virtuales de } \\
\text { aprendizaje. }\end{array}$ \\
\hline Autoevaluación & $\begin{array}{l}\text { No califica al estudiante cuantitativamente, sino que sirve como proceso } \\
\text { de reflexión para que el mida sus fortalezas y debilidades en el proceso de } \\
\text { aprendizaje. }\end{array}$ & $\begin{array}{l}\text { El docente solicita a cada estudiante un informe que rinda cuentas } \\
\text { de sus descubrimientos en términos de fortalezas y debilidades } \\
\text { de aprendizajes adquiridos. }\end{array}$ \\
\hline $\begin{array}{l}\text { Evaluación } \\
\text { en línea }\end{array}$ & $\begin{array}{l}\text { Los LMS disponen de esta herramienta infovirtual, diseñada para valorar los } \\
\text { aprendizajes adquiridos del estudiante, en forma cuantitativa y cualitativa. }\end{array}$ & $\begin{array}{l}\text { Dispone de preguntas y respuestas de selección múltiple, } \\
\text { apareamiento, desarrollo y respuesta calculada. El docente } \\
\text { realimenta la evaluación del estudiante, suscitándose un diálogo } \\
\text { virtual asincrónico. }\end{array}$ \\
\hline $\begin{array}{l}\text { Tareas y } \\
\text { actividades }\end{array}$ & $\begin{array}{l}\text { El docente programa ejercicios y actividades correspondientes al curso, } \\
\text { según la programación propuesta. }\end{array}$ & $\begin{array}{l}\text { El docente da las instrucciones precisas para que el estudiante } \\
\text { realice una actividad con un producto final, el cual es enviado } \\
\text { por el estudiante, retroalimentado y calificado por el docente, } \\
\text { generándose un diálogo virtual asincrónico. }\end{array}$ \\
\hline Los weblogs & $\begin{array}{l}\text { Hacen las veces de diario personal del usuario, presenta contenidos enrique- } \\
\text { cidos con hipervínculos, hipertextos, gráficos e imágenes que permiten al } \\
\text { interlocutor profundizar en las temáticas propuestas. }\end{array}$ & $\begin{array}{l}\text { El docente diseña estrategias que lleven al estudiante a } \\
\text { estudiar los contenidos y participaciones argumentadas del } \\
\text { blog. Finalmente, elige un representante para que recoja en un } \\
\text { documento resumen, las conclusiones y los aportes que ayudarán } \\
\text { a la construcción del aprendizaje de los estudiantes. }\end{array}$ \\
\hline
\end{tabular}

Fuente. Roldán, 2005, p. 69 
Como se observa, existen diversas herramientas que son utilizadas en la educación virtual, algunas de comunicación sincrónica y otras de comunicación asincrónica, que sirven para llevar a cabo el proceso de enseñanza aprendizaje, herramientas que le permiten al docente diseñar estrategias, $y$ al estudiante hacer un acercamiento a los conceptos y estudiar los contenidos. De igual manera, a ambos, docente vs. estudiante, la realización de un adecuado proceso de retroalimentación sobre la labor del estudiante que le permite a éste el logro de su aprendizaje y desarrollo de competencias.

Entre las estrategias formativas que sirven a la retroalimentación, se encuentran los espacios de discusión, los análisis de perspectivas, los ejercicios de comparación y los ejercicios de reflexión sobre lo evaluado; adicionalmente, también están los resúmenes, mapas conceptuales, ensayos, propuestas de investigación y el portafolio, los esquemas visuales de aprendizaje, las fichas de conceptualización, las técnicas de lectura, las técnicas de expresión, los cuadros comparativos y muchos otros (Maldonado, 2009).

Figura 3. Estrategias formativas que facilitan la retroalimentación

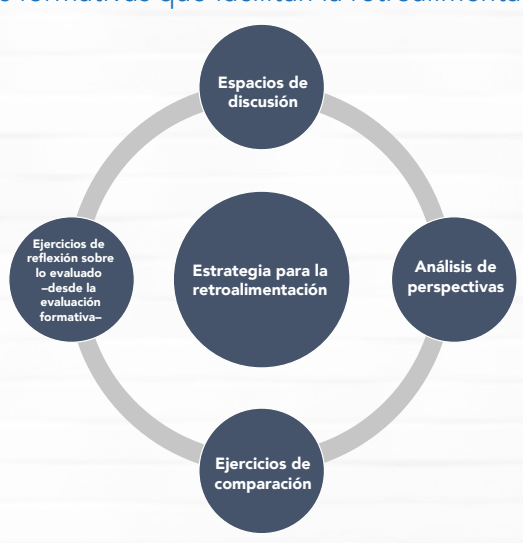

Fuente. Elaboración propia.
En pocas palabras, se podría decir que existen diversas herramientas virtuales que se utilizan para la retroalimentación y que el estudiante puede emplear en su proceso formativo y en su aprendizaje activo, pues, a través de ellas, comparte información, desarrolla la interacción grupal, puede visualizar diagramas, fomenta el trabajo cooperativo, aclara conceptos, se autoevalúa en términos de fortalezas y debilidades, transformándolo en un ser creador, dinamizador y autocrítico. También, estas herramientas promueven el diálogo entre el estudiante y el docente, quien a su vez crea estrategias formativas para obtener así la retroalimentación.

\section{Humanización del acto de la retroalimentación}

El punto de partida de esta sección se centra en el análisis sobre el concepto de humanización en el acto de la retroalimentación, en donde se pretende explicar los motivos que implica dicho proceso dentro del ambiente virtual.

Ellul (1990), en su libro La técnica o la apuesta del siglo, asevera que la tecnología es «la característica autónoma y definitoria de la sociedad moderna. Por ende, cuanto más avanza el progreso técnico, mayor será el carácter ético y espiritual que adquiera el problema social que conlleva este progreso» (p. 37). Al respecto, Criollo (2007) opina que esas reflexiones tanto éticas como espirituales no han avanzado al mismo tiempo que la tecnología, pues se ha presentado una ausencia que no permite encontrar de forma fácil cómo humanizar en y con la tecnología. 
Por el contrario, Mumford (2011), en su libro El mito de la máquina, hizo la invitación a humanizar la tecnología, resaltando que el hombre debe buscar su plenitud en aquello que le hace humano, como es en su inteligencia, en sus valores, en su crecimiento espiritual y en otros; por lo tanto, la tecnología debe estar armonizada con el mismo hombre y la naturaleza, y no que sea un instrumento de alienación y destrucción de su mundo.

Pasando al ámbito de la educación, Lozada et al. (2013) indican que la educación es la primera en ser requerida en todo proceso humanizador, debido a que asume al ser humano de forma integral para desarrollar todas sus potencialidades desde un contexto social y cultural. De esta manera, el binomio educador-educando debe actuar de manera holista, para mostrar así la forma como el educador puede ser, a su vez, educado por el alumno con el respeto mutuo entre el uno y el otro.

También expresa que la humanización en la educación es el acto de amor en lo que se hace, es apoyar, es inculcar valores, es no hacer uso del poder para reprimir, es llevar al estudiante a la libertad, al diálogo, a la reflexión y a la argumentación al decir lo que piensa, es aceptar las consecuencias en la toma de decisiones, es saber detectar el interés del estudiante, es despertar la curiosidad, la autonomía, sin descuidar el buen trato, el respeto, la amabilidad, la paciencia, la verdad, la justicia, el apoyo dentro y fuera del espacio académico, es afianzar su seguridad y su compromiso con un proyecto de vida propio que contribuya al bienestar con responsabilidad social (Lozada et al., 2013).
Por este motivo, los docentes, desde el marco instruccional, son los indicados para ubicar el desarrollo del aprendizaje desde la importancia del compromiso humano, del aprender a ser antes que del tener o saber, de encontrar cómo humanizar la tecnología, con el fin de interrelacionar el vértigo mediático que esta tiende a invadir, en cuanto al estudiante, debe tener capacidad para ser objetivo, escuchar pausadamente y de discernir de forma liberada, lo cual, debe ser propiciado y potenciado por el docente en la educación virtual (Criollo, 2007).

En la educación virtual, Criollo (2007) afirmó que prevalece una gran ventaja para asumir lo humano desde la virtualidad, a pesar de las carencias que existen por falta del contacto directo y es el acompañamiento individual que se hace al aprendizaje, que es de dos maneras: de forma cognitiva y de carácter humano, refiriéndose este último en inculcar valores, principios éticos, antropológicos, de trabajo de la condición humana, identidad regional, de proyecto de vida, de afectividad, de relaciones interpersonales y muchos otros.

Por este motivo, Bárcena (2000) opinó que a través de la educación se trata de exteriorizar lo humano que hay en cada persona, o lo que es lo mismo, se busca la humanización. Vargas (2006) estimó que «la tecnología potencia y realiza lo humano toda vez que pone el ingenio en escena y produce alteración del entorno de manera racional y positiva» (p. 150), lo que quiere decir que en los recursos tecnológicos que son utilizados para la retroalimentación, debe primar ante todo el criterio interaccionar y no el de uso tecnológico. 
En cuanto al criterio interaccionar, Roldán (2005) indicó que en la educación virtual se generan varias clases de interacciones que le apuestan a la humanización en el acto de la retroalimentación, estas son:

Tabla 3. Clases de interacciones

\begin{tabular}{|c|l|}
\hline $\begin{array}{c}\text { Interacción } \\
\text { Docente } \\
\text { facilitador-estudiante }\end{array}$ & $\begin{array}{l}\text { Actividades, procesos y mensajes afectivos y } \\
\text { motivadores que recíprocamente se generan } \\
\text { para retroalimentar conceptos, que mantienen } \\
\text { el flujo comunicativo en el proceso formativo. }\end{array}$ \\
\hline $\begin{array}{c}\text { Estudiante-materiales } \\
\text { didácticos o } \\
\text { contenidos }\end{array}$ & $\begin{array}{l}\text { Invoca la disciplina del estudiante, adoptando } \\
\text { técnicas de estudios para aprender de forma } \\
\text { autónoma, colaborativa y cooperativa. }\end{array}$ \\
\hline Estudiante-estudiante & $\begin{array}{l}\text { Trascienden las distancias físicas y establecen } \\
\text { relaciones como colegas y estudiosos, confor- } \\
\text { mando verdaderas comunidades académicas y } \\
\text { virtuales, donde la amistad y los intereses comu- } \\
\text { nes de conocimiento afloran continuamente. }\end{array}$ \\
\hline Estudiante-red & $\begin{array}{l}\text { Los estudiantes utilizan el internet y las bases } \\
\text { de datos para trascender, buscar, profundizar, } \\
\text { investigar, inferir, asimilar y aplicar en contexto } \\
\text { los diversos aprendizajes que adquieren. }\end{array}$ \\
\hline Estudiante-contexto & $\begin{array}{l}\text { Los aprendizajes adquiridos son aplicados en } \\
\text { los contextos respectivos de los estudiantes, así, } \\
\text { impacta positivamente el entorno social, cultural } \\
\text { y tradicional, para tener un conocimiento local } \\
\text { con trascendencia global. }\end{array}$ \\
\hline
\end{tabular}

Fuente. Roldán, 2005, p. 57.

Lo anterior se traduce a que las interacciones son procesos de comunicación que facilitan diferentes relaciones entre el estudiante con otros, con la tecnología y con las estrategias formativas. De este modo, obtienen la posibilidad de impactar, trascender y aprender de esa experiencia virtual, para así poder discernir entre lo humano y la tecnología. También, dentro de estas diferentes clases de interacciones, se visualiza la humanización en el ámbito virtual, porque se da la relación entre docente-estudiante; estudiante con otros estudiantes y el estudiante con comunidades en diferentes contextos.

Dentro de este marco, ha de considerarse el rol del docente en la educación virtual, pues una práctica docente humanizante se caracteriza por buscar una nueva forma de ser docente humanizador, por el compromiso que tiene de transformar el modelo tradicional en una práctica virtual. De esta manera, se busca lograr el desarrollo humano, formar en valores y ética, establecer relaciones académicas y personales equilibradas con el estudiante, enseñar para actuar con parámetros humanizantes, donde haya actitudes de humildad, respeto, confianza, responsabilidad, tolerancia y otros, que le permiten unas habilidades, como son el pensamiento crítico-reflexivo, autónomo, con conocimientos multidisciplinares, que contribuyen al incremento de aprendizajes significativos para el estudiante, que lo van a formar como profesional humanizado (Lozada, et al., 2013).

Lo anterior señala que el primer indicio de humanización que se da en la educación virtual está en el compromiso que tiene el docente de lograr el desarrollo humano del estudiante, en sus diferentes aspectos de formación. Al respecto, Merayo (2000) citado por Criollo (2007), argumentó que el docente tiene grandes retos en la educación virtual, que se orientan en varias direcciones: a) enseñar a buscar, con el fin de poder investigar y distinguir lo menos a lo más importante, en una oferta de información que excede la capacidad de 
asimilación de la inteligencia humana, b) enseñar a entender, para captar la esencia de los conceptos y encontrar nuevas relaciones entre ellos, obtener conclusiones y así relacionar causas y consecuencias, para así lograr mejoras cualitativas y cuantitativas de las estructuras cognitivas que ya se posee, c) enseñar a aplicar el sentido crítico, como norma en la construcción del conocimiento y en todas las conductas vitales y d) enseñar a comunicar y a expresar las propias ideas, en un marco abierto al diálogo y al respeto mutuo, todo ello para retroalimentar como componente principal de la interacción.

Una vez más, se deduce que la humanización en el acto de la retroalimentación se refleja en todo el proceso que se da en la formación virtual, en donde el docente debe realizar diversas actividades para que el estudiante logre su objetivo de aprendizaje. Por esta razón, la retroalimentación en la educación virtual se ha considerado primordial, por dos aspectos. En primer lugar, fortalece vínculos entre alumno y tutor y establece comunicación sincera con un gran contenido ético frente al desempeño y rendimiento del estudiante en su proceso educativo (Pinilla, 2016). En segundo lugar, representa una conversación con el estudiante como parte del ambiente formativo, y dicha conversación tiene como misión generar cambios, modificar diversas formas de producir los trabajos, de interpretar las finalidades educativas, asegurar la meta pretendida dentro del perfil de egreso de un programa educativo, actividad que se realiza a través del uso de una importante herramienta cultural, base del aprendizaje que es el lenguaje, con el cual el docente ayuda al alumno a crecer haciéndolo consciente de quién es mediante lo que él realiza (Martínez, 2011).
En suma, por el clima de convivencia, compromiso y pertenencia que se desarrolla en la construcción de la virtualidad, uno de los primeros indicios de humanización que se reflejan en el acto de la retroalimentación, es la dedicación que debe tener el docente con el estudiante. Un segundo indicio es todo el proceso que se desencadena dentro del acto de la retroalimentación entre el docente y el alumno, que se evidencia en el seguimiento que se hace al estudiante, el conocimiento que se tiene de sus dificultades, el progreso que se da a su aprendizaje y en la identificación de su contexto.

\section{Conclusiones}

La retroalimentación es el principal mecanismo de aprendizaje, debido a que en la educación virtual las actividades realizadas son documentos escritos para ser leídos, esto hace que muestre una lectura seria por parte del docente, quien está obligado a dejar evidencia de dicho acto, lo que constituye una comunicación entre el docente y el estudiante, de ahí que el vínculo que se da entre las dos partes en un ambiente virtual se fortalece a través de adecuados procesos de retroalimentación, que debe estar regulada para que la formación virtual cumpla las metas de una verdadera educación, porque de ella depende que el estudiante crea y tenga confianza en sí mismo, le genere reflexión, alimente su autoestima, lo motive y le refuerce y acreciente su aprendizaje.

Existen diversas herramientas virtuales que se utilizan para la retroalimentación y que son utilizadas por el estudiante en su proceso formativo y en su aprendizaje activo, pues a 
través de ellas comparte información, desarrolla la interacción grupal, puede visualizar diagramas, fomenta el trabajo cooperativo, aclara conceptos y se autoevalúa en términos de fortalezas y debilidades. De este modo, se transforma en un ser creador, dinamizador y autocrítico, también estas herramientas promueven el diálogo entre el estudiante y el docente, quien a su vez crea estrategias formativas para obtener así la retroalimentación.

Por el clima de convivencia, compromiso y pertenencia que se desarrolla en la construcción de la virtualidad, uno de los primeros indicios de humanización que se reflejan en el acto de la retroalimentación es la dedicación que debe tener el docente con el estudiante, un segundo indicio es todo el proceso que se desencadena dentro del acto de la retroalimentación entre el docente y el alumno, que se evidencia en el seguimiento que se hace al estudiante, el conocimiento que se tiene de sus dificultades, el progreso que se da a su aprendizaje y en la identificación de su contexto.

\section{Referencias}

Ávila, P. (2009). La importancia de la retroalimentación en los procesos de evaluación. Una revisión del estado del arte. Universidad del Valle de México: México. Recuperado de https://www.educar.ec/servicios/0-Avila retroalimentacion. pdf

Alvarado, M. A. (2014). Retroalimentación en educación en línea: una estrategia para la construcción del conocimiento. Revista RIED, 17(2), 59-73. DOI: https://doi.org/10.5944/ ried.17.2.12678
Bárcena, F. (2000). El aprendizaje como acontecimiento ético. Sobre las formas del aprender. Enrahonar, 31, 9-33. Madrid. Recuperado de http://bit.ly/2kY3E1Z

Carbonell, E.; Hortolà, P, (2013). Hominización y humanización, dos conceptos clave para entender nuestra especie. Revista Atlántica-Mediterránea, 15, 7-11. Recuperado de http://bit. ly/2ko20pY

Cardona, H. E. (2008). Consideraciones acerca de la educación virtual como comunidad de relaciones afectivo-valorativas. Revista Iberoamericana de Educación, 46(7), 10.

Criollo, F. A. (2007). Potenciar lo humano en la educación virtual. Recuperado de http://bit.ly/2kXhjWT

Cruz, M. L. (2008). Evaluación formativa y autorregulación. Un estudio de caso. (Tesis de maestría). Universidad San Francisco de Quito. Quito, Ecuador. Recuperado de http://bit.ly/2mrAHMf

Durante, I.; Sánchez, M. (2006). La retroalimentación en la educación médica. Ponencia presentada en el Seminario: el ejercicio actual de la Medicina. Universidad Nacional Autónoma de México, Ciudad de México, México, septiembre de 2006.

E-learning Masters. (2018). El aula semipresencial y sus beneficios. Recuperado de http://bit.ly/2mbjtnf

Ellul, J. (1990). La technique ou l'enjeu du siècle. París: Économica.

Fornells,J.M.; Juliá,X.;Arnau,J.;Martínez-Carretero, J.M.(2008). Feedback en educación médica. Educación Médica, 11(1), 7-12.

Gitman, L. J.; MacDaniel, C. (2008). El futuro de los negocios. Quinta edición. México: CENGAGE Learning. 
Lozada, O. P.; Beltrán, O. M.; Vargas, F. A.; Martín, D. A.; Hincapié, B. S.; Herrera, M.; Pérez, B. A. (2013). Humanización de la práctica docente universitaria. Bogotá. Recuperado de http:// bit.ly/2ksg81w

Maldonado, C. R. (2009). Sobre la retroalimentación o el feedback en la educación superior online. Revista Virtual Universidad Católica del Norte, 26, 1-18. Recuperado de http://bit. ly/2ksgtBk

Martínez, P. L. (2011). Reflexiones sobre la retroalimentación en ambientes virtuales. Ponencia presentada en el II Encuentro Virtual Educa. Instituto Tecnológico de Estudios Superiores de Monterrey. Monterrey, México, 29 de abril.

Ministerio de Educación Nacional. (2009). Educación Superior o Educación en línea. ¿Qué es la educación virtual? Recuperado de http://bit.ly/2kLiOTo

Mogollón, I. (2004). El chat y otros procedimientos de evaluación a distancia aplicables en sistemas mixtos. Pixel-Bit Revista de Medios y Educación, (23), 43-54.

Mosley, D. C.; Megginson, L. C.; Pietri, P. H. (2005). Supervisión: la práctica del empowerment, desarrollo de equipos de trabajo y su motivación. $6^{a}$ edición. Grupo GEO Impresores: Madrid, España.

Mumford, L. (2011). El pentágono del poder. El mito de la máquina. España: Editorial Pepitas de Calabaza.

Osorio, K.; López, A. (2014). La retroalimentación formativa en el proceso de enseñanza aprendizaje de estudiantes en edad preescolar. Revista Iberoamericana de Evaluación Educativa, $7(1), 13-30$
Papic V.; Rittershaussen, K. S.; Rodríguez, R. E. (1987). Importancia de la retroalimentación en el desarrollo de habilidades docentes de comunicación. Revista Electrónica Tecnológica y Comunicación Educativas, 3(6). Recuperado de http://bit. ly/2IYwT51

Parra, J. E. (2005). Aproximación a la virtualidad desde la propuesta educativa de la Fundación Universitaria Católica del Norte (FUCN). Educación virtual: reflexiones y experiencias. FUCN. Recuperado de http://bit.ly/2m0iZiE

Amaranti, M. (2010). Evaluación de la educación: concepciones prácticas de retroalimentación de los profesores de lenguaje y comunicación de primer año de educación media investigación educativa con estudio de caso. Ponencia presentada en el Congreso Iberoamericano de Educación Metas 2021. Buenos Aires, Argentina, 13-15 septiembre. Recuperado de http://bit.ly/2ksgFAy

Pérez, J.; Salas, M. (2016). Características de la retroalimentación como parte de la estrategia evaluativa durante el proceso de enseñanza aprendizaje en entornos virtuales: una perspectiva teórica. Revista Calidad en la Educación Superior, 7(1), 175204.

Pérez, M.; Subirà, M.; Guitert, M. (2011). Aprender y enseñar en línea. Recuperado de http://bit.ly/2m6wfSI

Pinilla, J. C. (2016). Herramientas de apoyo a la retroalimentación en EVA. (Trabajo de maestría). Universidad Cuauhtémoc. Bogotá, Colombia. Recuperado de http://bit.ly/2mmX4Cl

Ralón, L., Vieta, M., y Vásquez, M. L. (2004). (De)formación en línea: acerca de las desventajas de la educación virtual. Comunicar, Revista Científica de Comunicación y Educación, 22, 171176. Recuperado de http://bit.ly/2ksrlPD 
Roldán, N. D. (2005). Comunicación y pedagogía para el arte de aprender. Educación virtual: reflexiones y experiencias. FUCN. Recuperado de http://bit.ly/2mktVaW

Rubio, Á. L. (2002). Internet y enseñanza: la educación virtual. Universidad Complutense de Madrid. Facultad de Ciencias de la información. Recuperado de http://bit.ly/2mtpc7b

Scagnoli, N. (2001). El aula virtual: usos y elementos que la componen. Recuperado de http://bit.ly/2kM9CD9

The flipped classroom. (2017). Clase invertida. Recuperado de http://bit.ly/2m2jsRp

Unigarro, M. A. (2001). Procesos críticos en la evaluación virtual. Bucaramanga: Educación Virtual: encuentro formativo en el ciber espacio.

Vargas, G. (2006). Tratado de epistemología. Fenomenología de la ciencia, la tecnología y la investigación social. Bogotá: San Pablo.

Villardón, L. (2006). Evaluación del aprendizaje para promover el desarrollo de competencias. Educación Siglo XXI, Universidad de Deusto. 